\title{
Vibration Signal Denoising using Neighbourhood and Parent-Child Relationship of Wavelet Transform Coefficients
}

\author{
Pooja Yadav \\ Dept. of Electronics and Communication \\ S A T I Vidisha (MP), INDIA
}

\begin{abstract}
A method based on intra-scale and inter-scale dependency of coefficient of stationary wavelet transform has been developed for vibration signal denoising. In this paper, features of Stationary Wavelet Transform are revised by comparing it to Discrete Wavelet Transform. Proposed denoising method is simulated for different noise values and results are compared to other denoising methods. Proposed method is used for treatment of practical signals to confirm that the proposed method is suitable and efficient in improving the SNR of the vibration signal and in processing the original information by retaining its shape.
\end{abstract}

\section{General Terms}

DWT, SWT, Thresholding

\section{Keywords}

De-noising, Vibration signal, Wavelet Transform, Parentchild relationship, Neighbourhood

\section{INTRODUCTION}

Machines play important role in speeding up the production of modern manufacturing industries. Production of an industry depends on the efficiency of machines. Hence maintenance of machinery system is important. Rotary machine is a tool that contains internal moving parts such as gear, bearing, rotor etc. These rotating parts may get damaged due to excess loading or stress and may develop fault [1]. Detection of faults can be done by analysing the vibration signal, but during the acquisition of vibration signal, it get contaminated with external noise and denoising of vibration signal becomes essential. Many techniques have been developed for vibration signal denoising that are based on Fourier Transform (FT). However FT is not suitable for nonstationary signals [2-3]. Short time Fourier Transform (STFT) overcomes the drawback of FT but it creates constant resolution for a window and is suitable for quasistationary signals and not for pure non stationary signals [4]. Technique based on empirical mode decomposition [5], developed for non-stationary signals, decomposes signals into small intrinsic mode functions but these functions are not purely orthogonal which may produce end effects and may become the reason of mode mixing. Techniques based on wavelet transform have been developed that give better time-frequency resolution of non-stationary signals. Discrete wavelet Transform (DWT) is one of the fastest techniques [6] that analyses the signal by filtering and subsampling. At the reconstruction stage, reverse process is done but it has downside of being shift variant because DWT coefficient of shift shifted version of original signal are not identical to the coefficients of the delayed signal [7].The Stationary Wavelet Transform

\author{
Preety D. Swami \\ Dept. of Electronics and Instrumentation \\ S A T I Vidisha (MP), INDIA
}

(SWT) overcomes the drawback of DWT as it is shift invariant [8]. Many thresholding methods have been proposed earlier such as term by term thresholding [9],in which each wavelet coefficient was compared to a predetermined threshold value and then the coefficient value was changed by either hard thresholding or soft thresholding. In local block thresholding, rather than comparing individual coefficient, a group of coefficients was compared [10-12] with a threshold value. Wavelet coefficients have not only intrascale dependency but interscale dependency too [13]. In this paper a denoising method has been proposed in which a wavelet coefficient is thresholded on the basis of its relationship with its neighbours and parent. In Section II, a brief introduction to SWT and its comparison to other techniques is given. Proposed denoising method is discussed in Section III. Results by applying proposed method to practical signal and comparison of results with that of other techniques are mentioned in Section IV. Finally conclusions are drawn in Section V.

\section{STATIONARY WAVELET TRANSFORM}

In this section features of SWT are explored. SWT was introduced in 1996 and is popular because of its shiftinvariance nature. It is a modified version of DWT in which the interpolation and decimation filters have been interpolated [8]. Instead, zero padding was done at each filter level with its shift invariant property; it has enhanced the applications of wavelet transform for denoising purposes. Using SWT, the signal is decomposed by passing original signal through sets of high pass and low pass filters. These filters produces output data sequences of same length as that of the original data sequence. If $a^{j}$ are original sequence then approximation and detail coefficient sequences can be calculated as:

$$
\begin{aligned}
& a^{j-1}=H^{|j-1|} a^{j} \\
& b^{j-1}=G^{|j-1|} a^{j}
\end{aligned}
$$

If $a^{j}$ is of length $2^{j}$ then $a^{j-1}$ and $b^{j-1}$ will be of length $2^{j}$ instead of being shorter, as level increases. $H^{r}$ And $G^{r}$ are sets of filter at each level. Zeros are added adjacent of each element of filter of the earlier stage. At reconstruction stage, decomposed signal is passed through the sets of inverse filters $H^{-1}$ and $G^{-1}$ for reconstruction of the original signal.

\section{PROPOSED WORK}

In this section DWT has been compared to SWT. The demonstration of the superiority of SWT over DWT is done through analysis of frequency spectrum of multiple harmonic signals. Decomposition of multiple harmonic signals is done both by DWT and SWT. The wavelet db8 
is used for both DWT and SWT decomposition. It can be easily observed that DWT experience shift variance which does not happen with SWT. To explore this we generate a signal, $s_{1(t)}$ of sampling frequency $2000 \mathrm{~Hz}$ :

$$
\begin{aligned}
s_{1(t)}= & .5 \cos (2 \pi .30 t)+\cos (2 \pi .150 t)+1.5 \cos (2 \pi .200 t) \\
& +\cos (2 \pi .300 t)+.5 \cos (2 \pi .500 t)
\end{aligned}
$$

Where $t \in[0,0.256]$. Above mentioned signal consists of five frequency components:30, 150, 200, 300 and $500 \mathrm{~Hz}$. Decomposed signal using DWT and SWT is shown in Fig.1(a) and Fig.1(b) and their frequency spectrum is obtained by taking FFT of decomposed signal that are shown in Fig.1(c) and Fig.1(d) respectively. By analysing FFTs of the decomposed levels by DWT it is observed that it contains unwanted frequency components of $100 \mathrm{~Hz}$ and $700 \mathrm{~Hz}$ at detail levels D4, D3 and D1 that does not exist in the original signal. Moreover FFTs of the decomposed levels of SWT does not produce or contain any frequency component other than $30,150,200,300,500 \mathrm{~Hz}$ at different decomposition levels. This shows SWT is better than DWT for signal spectrum analysis and more suitable for denoising of non-stationary signals.

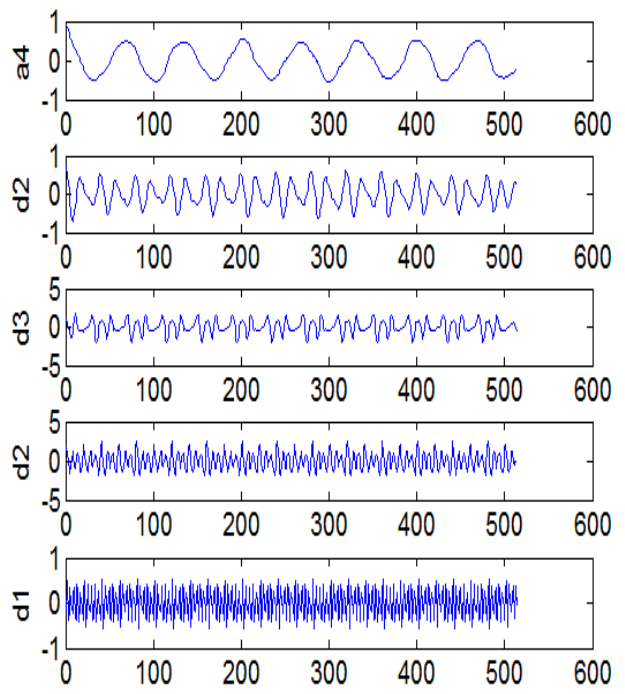

(a)

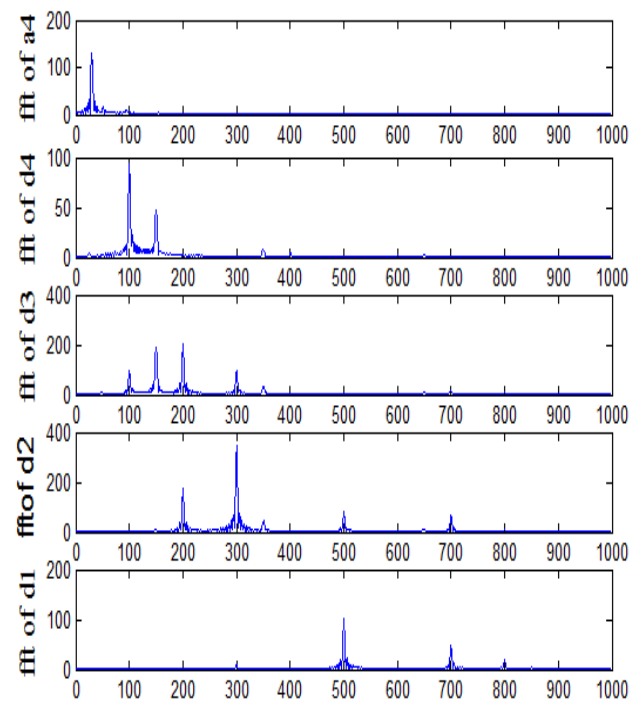

(b)

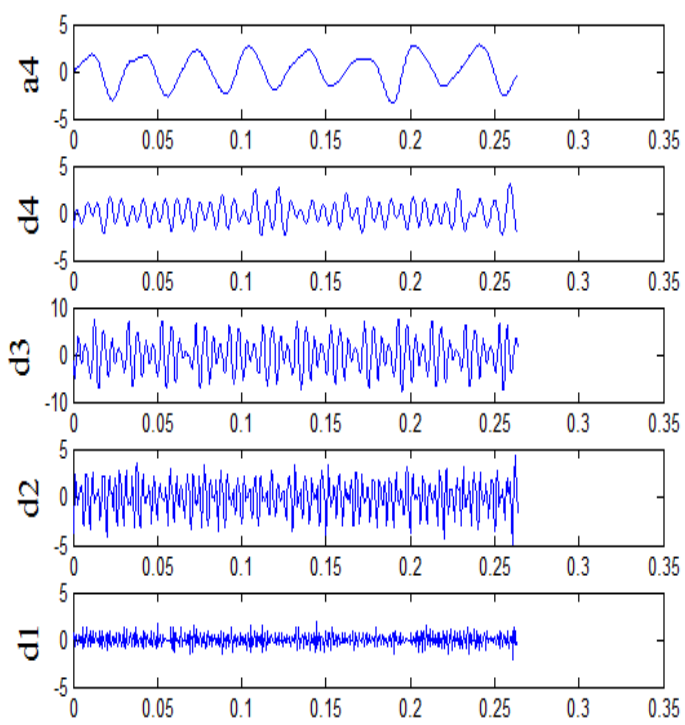

(c)

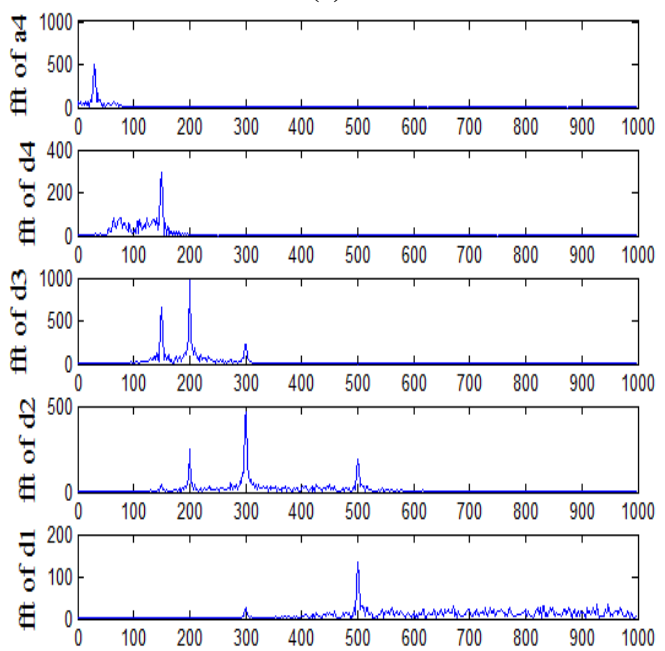

(d)

Fig.1(a) DWT decomposition of multiharmonic signal (b) FFT of decomposed signal using DWT (c) SWT decomposition of multiharmonic signal (d) FFT of decomposed signal using SWT

\subsection{Proposed Denoising Method}

It is observed that denoising can be improved by block thresholding of wavelet coefficients as compared to term by term thresholding of these coefficients. Averaging window technique is also based on block thresholding but it considers only intrascale dependency of wavelet coefficients.

If we consider the parent coefficients along with the neighbour coefficients it improves the SNR of the reconstructed signal. Basic idea of the proposed denoising method is taken from the fact that ; wavelet coefficients are correlated with their neighbours as well as with their parent coefficients as seen in Fig.2. But the noisy coefficients are neither correlated with neighbours nor with the parent coefficient. The proposed denoising method involves the following steps: 
3.1.1 Decomposition of original signal using SWT to produce approximation and detail sequences of the input noisy signal.

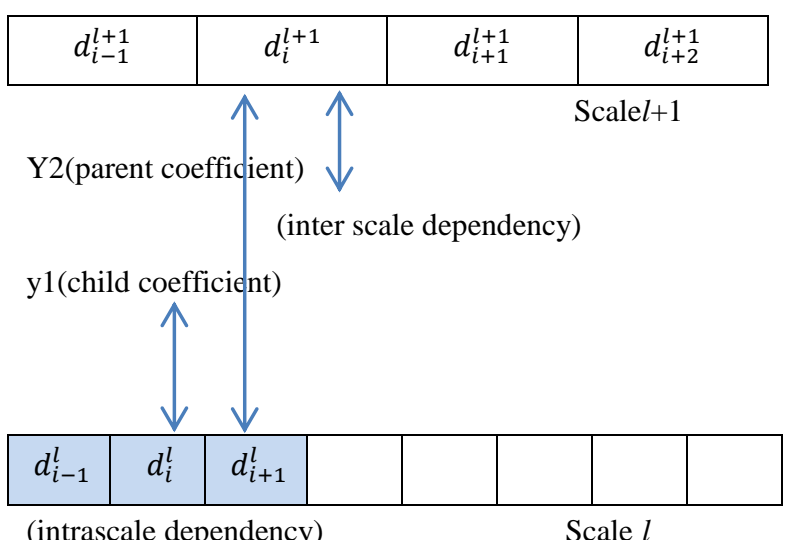

Fig. 2 Correlation of wavelet coefficients

3.1.2 Detail coefficients contain high frequency components which belong to noisy coefficients. Hence preprocessing is done on detailed coefficients. [3]

3.1.3 If $l$ defines different levels then we compute the following parameters:

$$
S_{l i}^{2}=d_{i-1}^{l}+d_{i}^{l}+d_{i+1}^{l}
$$

Where $d_{i}^{l}$ is detailed coefficient to be thresholded and $d_{i-1}^{l} \& d_{i+1}^{l}$ are its neighbours

$$
S_{l+1 i}^{2}=d_{i-1}^{l+1}+d_{i}^{l+1}+d_{i+1}^{l+1}
$$

Where $d_{i}^{l+1}$ is the parent coefficient of $d_{i}^{l}$ and $d_{i-1}^{l+1} \&$ $d_{i+1}^{l+1}$ are neighbours of $d_{i}^{l+1}$

$$
\begin{gathered}
\mathrm{S}_{\mathrm{i}}=S_{l i}^{2}+S_{l+1 i}^{2} \\
\lambda_{l}=\sigma^{l} \sqrt[2]{\log _{2} N} \\
\lambda_{l+1}=\sigma^{l+1} \sqrt[2]{\log _{2} N} \\
d_{i}^{l}=d_{i}^{l}\left(1-\left({\lambda_{l}}^{2}+\lambda_{l+1}^{2}\right) /\left(S_{l i}^{2}+S_{l+1 i}^{2}\right)\right.
\end{gathered}
$$

3.1.4 $S_{i}$ is compared to the threshold value $\left(\lambda_{l}\right.$ or $\left.\lambda_{l+1}\right)$ : if $S_{i}$ is less than the threshold value, then the detail coefficient is set to zero else it is thresholded using soft thresholding, expressed by (9). Method is repeated for each level. In this adaptive threshold method, threshold value depends on coefficients at the levels $l$ and $l+1$.

3.1.5 Inverse SWT is taken for the reconstruction of the denoised signal.

\section{EXPERIMENTS AND RESULT}

\subsection{Denoising of Simulated Signal}

For simulation of proposed denoising method we create a signal similar to faulty gear signal consisting of periodic impulses of period $0.12 \mathrm{~s}$ sampled at $2000 \mathrm{~Hz}$, expressed by following equation.

$s_{2}(\mathrm{t})=.4 \sum_{i=1}^{4} \delta(-111 i)+\sum_{i=1}^{4} \operatorname{rand}(1) \exp (-.12(t-$ $\left.\left.t^{\prime}\right)\right) \sin \left(2 \pi 400 t^{\prime \prime}\right)$
Simulated signal is shown in Fig. 3(a). Noise of distinct standard deviation is added to the simulated signal is shown in Fig. 3(b). Noisy signal is decomposed at decomposition levels using db8 wavelet. Proposed denoising method was applied to the detail coefficients of the noisy signal to eliminate noise. Denoised signal using the proposed method is shown in Fig.3(e). Averaging window with hard thresholding and soft thresholding, are shown in Fig.3(c) and Fig.3(d) respectively, also have been applied to compare the results. For comparison we have used the parameter SNR which compares the ratio of signal power to noise power. Results extracted by averaging window with hard thresholding, soft thresholding and with proposed denoising method is shown in table 1 .

Table 1 Comparison of output SNR observed by using SWT with averaging window technique [12] and SWT with proposed denoising method

$\boldsymbol{\sigma}$-Standard Deviation

\begin{tabular}{|c|c|c|c|c|}
\hline \multirow{2}{*}{$\sigma$} & \multirow{3}{*}{$\begin{array}{c}|c| \\
\text { Onput } \\
\text { SNR }\end{array}$} & \multicolumn{2}{|c|}{$\begin{array}{c}\text { Output } \\
\text { (avg. window) }\end{array}$} & $\begin{array}{c}\text { SNR } \\
\text { (proposed } \\
\text { denoising } \\
\text { method) }\end{array}$ \\
\cline { 3 - 5 } & & $\begin{array}{c}\text { Hard } \\
\text { Thresholding }\end{array}$ & $\begin{array}{c}\text { Soft } \\
\text { Thresholding }\end{array}$ & $\begin{array}{c}\text { Soft } \\
\text { Thresholding }\end{array}$ \\
\hline 0.01 & 18.8790 & 16.5283 & 22.9302 & 23.0071 \\
\hline 0.02 & 12.8584 & 12.6512 & 17.7074 & $18 . .0641$ \\
\hline 0.03 & 9.3366 & 10.3523 & 15.0814 & 15.0947 \\
\hline 0.04 & 6.8378 & 8.7627 & 12.8714 & 13.0777 \\
\hline 0.05 & 4.8996 & 7.5949 & 11.0339 & 11.4582 \\
\hline 0.06 & 3.316 & 6.5787 & 9.2729 & 10.0152 \\
\hline 0.07 & 1.9770 & 5.7493 & 7.6223 & 8.7900 \\
\hline 0.08 & 0.8172 & 5.0440 & 6.6214 & 7.7263 \\
\hline
\end{tabular}

\subsection{Denoising of Practical Faulty Signal}

For examining the efficiency of proposed denoising method to real time faulty signals, we acquired signal from CWRE bearing data centre that contains fault in inner race at fan end side and sampled at $12000 \mathrm{~Hz}$, shown in Fig 4(a). Diameter of inner race is .007 inches and load is 1HP. Signal denoised by SWT using averaging window technique with hard thresholding shown in Fig.4 (b). and the noisy signal denoised by proposed method is shown in fig.4(c). From the comparison of fig.4 (b) and Fig.4(c) it is clearly visible that signal denoised using averaging window technique reduces noise but it eliminates original information too by suppressing the impulses present in the original signal. On the other hand results of proposed denoising signal not only reduce the noise but retain the shape of original signal maintaining its periodic impulses, which are a major source for fault detection. 

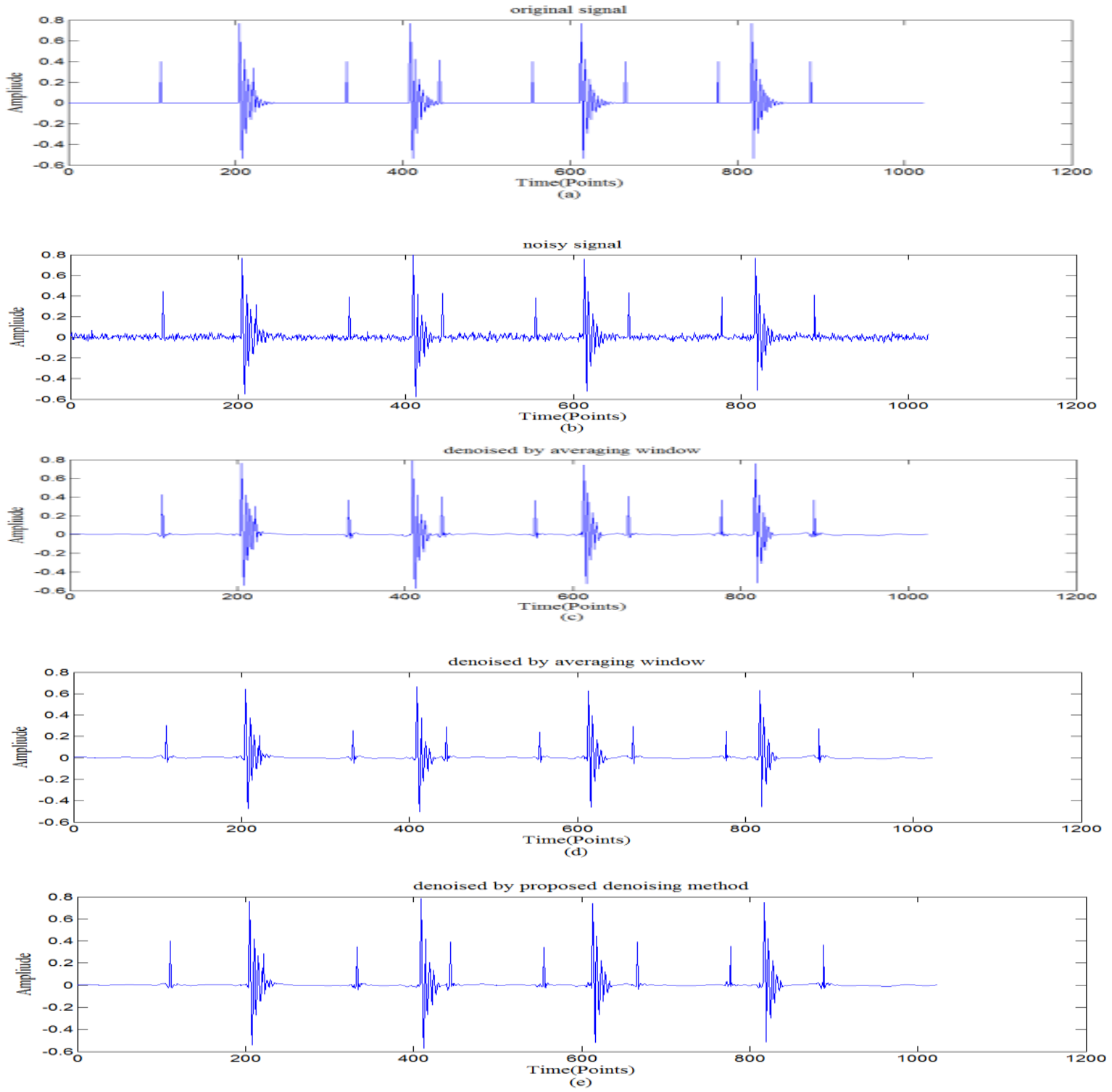

Fig.3: (a) Simulated faulty signal (b) Noisy signal with standard deviation 0.02 (c) Denoised signal using averaging window technique with hard thresholding (d) Denoised signal using averaging Window technique with soft thresholding. (e) Denoised signal using Proposed denoising method

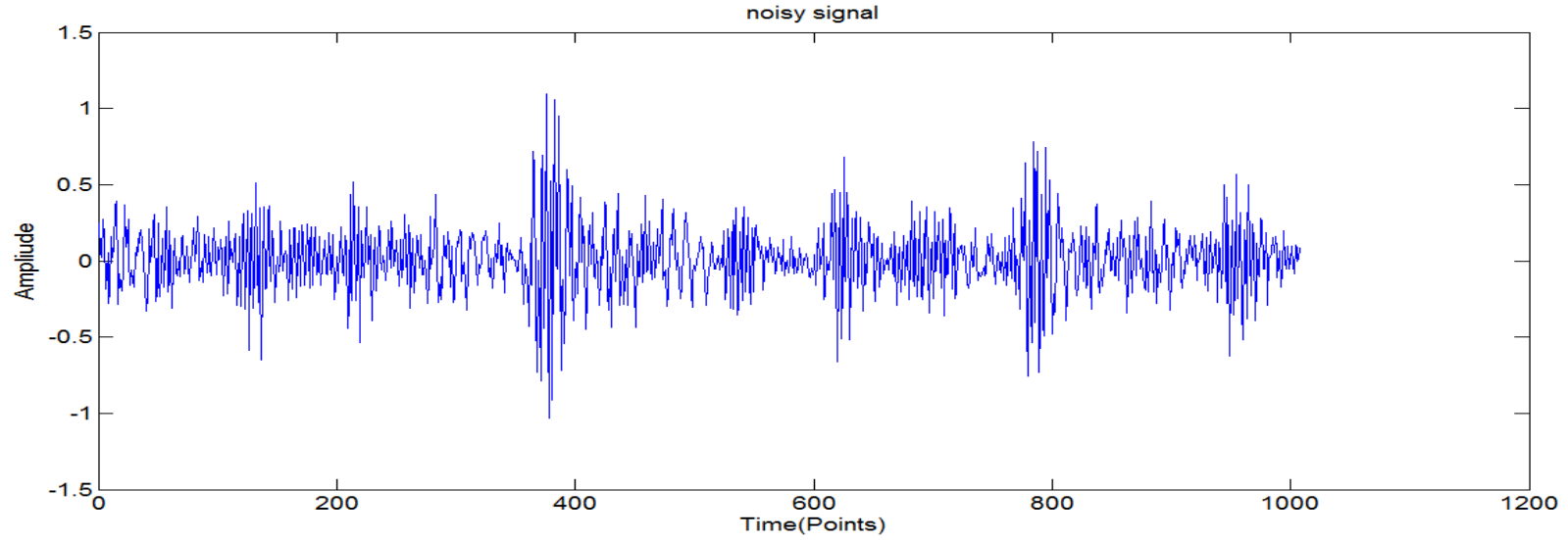

(a) 


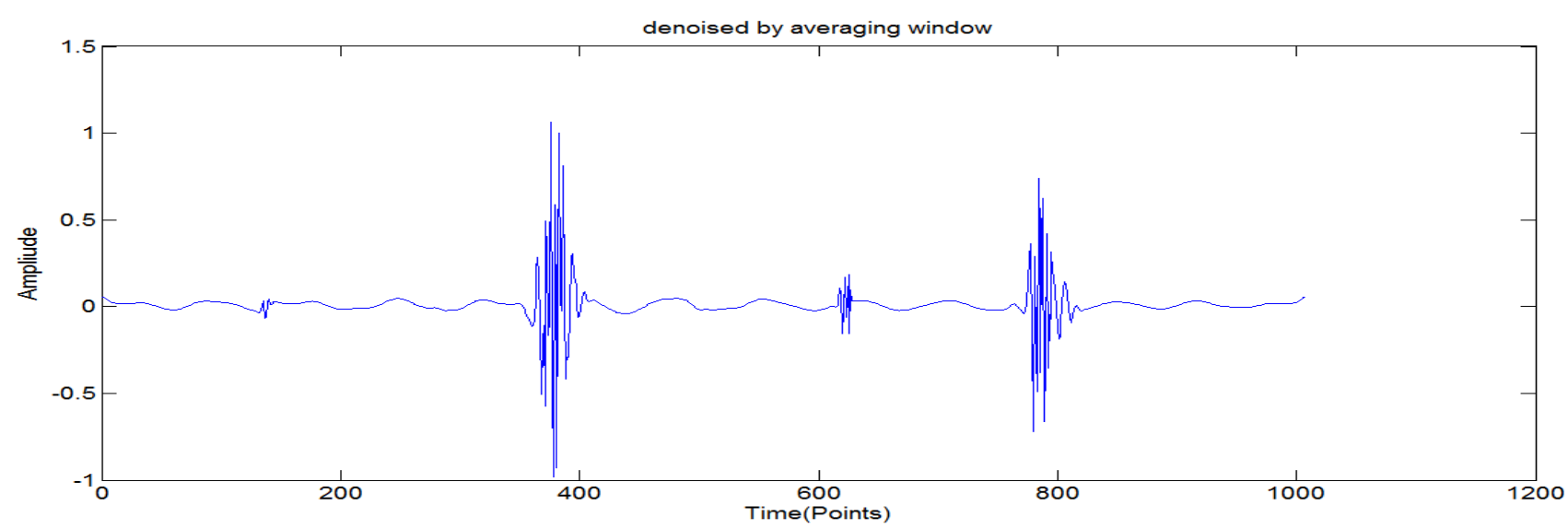

(b)

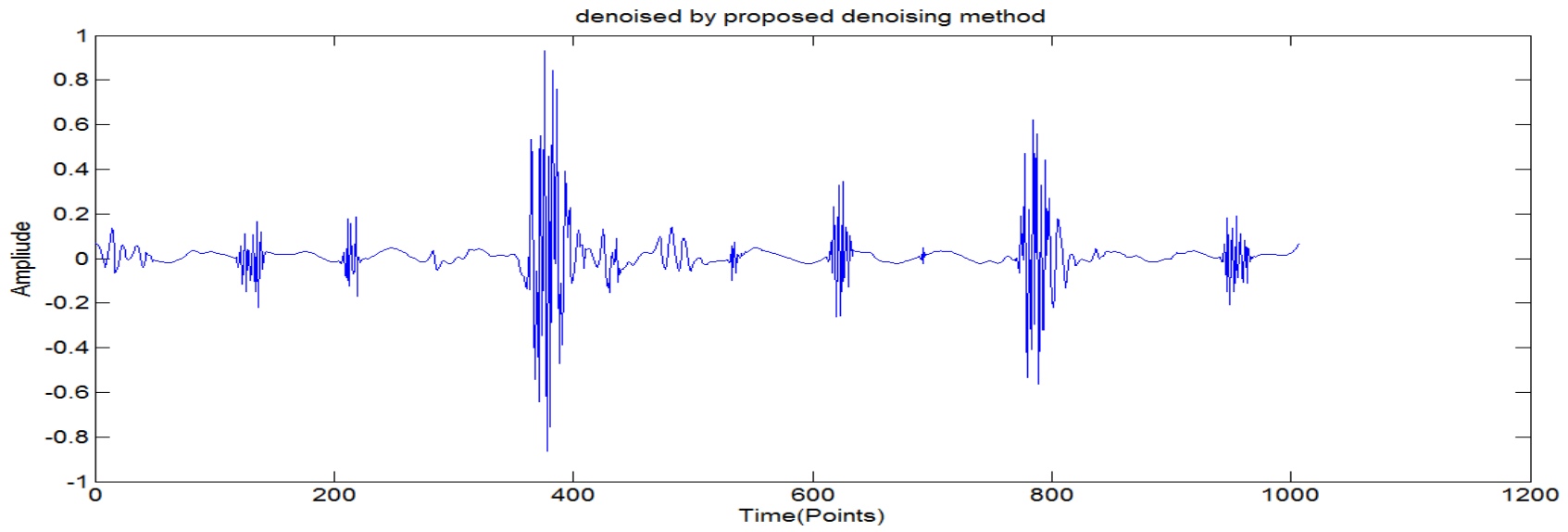

(c)

Fig.4: (a) Noisy signal fault at inner race (b) Denoised signal using averaging window technique with hard thresholding (c) Denoised signal using averaging

\section{CONCLUSION}

In this paper signal denoising method based on neighbour and parent child dependency of the wavelet coefficients has been proposed. This process was examined by applying it to noise like signal and results were compared to averaging window technique of denoising. By applying both the methods to practical signal, it is observed that the proposed denoising method removes noise more effectively and the denoised signal retains original shape of signal after eliminating the noisy content. It is also observed that if noise level increases proposed method gives better results. In future, better results can be expected by applying the proposed adaptive thresholding technique by analyzing the detail coefficients and varying the thresholds based on some specific statistical parameter.

\section{REFERENCES}

[1] B.K.N. Rao, Handbook of Condition Monitoring, Elsevier Advanced Technology, Oxford, 1996.

[2] H. Mahguon, R.E. Bekka, and A. felkaoui, "Gearbox fault detection using a new denoising method on ensemble emphirical mde decomposition and FFT," Mechanical System and Signal Processing, vol. 11,no. 3,pp.331-350 .
[3]. Polikar "The wavelet tutorial by Robi Polikar ." Avalable:http://users.rowan.edu/ polikar/WAVELET S/WTtutorial.html,1996.

[4] Z.K. Peng, F.I. Chu "Application of wavelet Transform in Machine Condition Monitoring and Fault Diagnostic: A review with biblography,Mech sys.Signal process 18(2004) 199-221.

[5] Yaguo Lei , Jing Lin , Zhengjia He, Ming J. Zuo , “A review on empirical ode decomposition in fault diagnosis of rotating machinery" oct.2012.

[6] J.Antonino-Daviu,M. Riera-Guasp,J. Roger-Folch, F.Martinez-Gimenez,A.peris "Application and optimization of discrete wavelet transform for detection of broken rotor bars in induction machines" March 2006.

[7] R.Rubini, and U.Meneghetti, "Application of the envelope and wavelet transform anaysis for the diagnosis of incipient faults in ball bearings," Mechanical System and Signal Processing, vol. 15 no. 2 , pp. 287-302, March 2001

[8] G.P. Nason and B.W. Silverman " The Stationary Wavelet Transform and some Statistical Application" in: Department of Mathematics, University of Bristol, Bristol BS8 ITW , UK, 1996. 
[9] D.L. Donoho , Denoising by soft thresholding, IEEE transaction on information theory 41(1995) 613-627.

[10] R.R. Coifman, D.L. Donoho, Translation invariant denoising, in: wavelet and statistics, Springer Lecture Notes in Statistics Vol. 103, Springer, New York, pp. 125-150.

[11] T.T. Cai, B.W. Silverman, Incorporating information on neighbouring coefficients in wavelet estimation, Sankhya: The Indian Journal of statistics Series B 63 (Part 2) (2001) 127-150.
[12] Shweta Garde,Preety D. Swami “ Noise Reduction of Vibration Signals in Rotary Machines using Neighbourhood Correlation of Wavelet Transform Coefficient", in: International Conference on Signal Processing, Embedded System and Commnication Technologies annd their application for Sustainable and Renewable Energy, June 2014.

[13] Li Zhen, He Zhengjia, Zi Yanyang\#, Wang Yanxue\# "Cutomized wavelet denoising using intra and inter scale dependency for bearing fault detection" school of mechanical engineering, Xian Jiaotong University, 710049, China, accepted 17 November 2007. 